\title{
COMENTARIO EDITORIAL: Inequidad en salud y educación: análisis introductorio de cara a un modelo de país
}

\author{
Health inequalities and education: introductory analysis on face a country model
}

Diego Bernardini*, Fernando Pedrosa**

\begin{abstract}
Resumen
La inequidad, la salud y la educación son tres indicadores relacionadas que condicionan el desarrollo de una sociedad. En este artículo se intenta hacer una aproximación a su mejor comprensión de cara a la planificación y gestión intersectorial como una de las bases que hacen a un modelo de país.
\end{abstract}

\section{Abstract}

Inequity, health and education are three related indicators that determine the development of the society. This paper offers an approach to better understand their interaction with planning and inter-sectorial management, as a foundation of a country model.

Palabras clave: inequidad, salud, educación, desarrollo social. Key words: inequity, health, education, social development.

Bernardini D, Pedrosa F. Inequidad en salud y educación Análisis introductorio de cara a un modelo de país. Evid. Actual. Práct. Ambul; 12(1): 2-3, Ene-Mar 2009.

\section{Intoducción}

Cuando se publicaron los resultados del Informe PISA 2006 (Programa para la Evaluación Internacional de los Estudiantes; del inglés: Programme for International Student Assessment) los cuestionamientos sobre el sistema educativo volvieron a estar en la agenda. Este relevamiento, que es realizado desde el año 2000 por la Organización para la Cooperación y el Desarrollo Económico (OCDE) mide las distintas competencias de los sistemas educativos de los países.

Además de sus 30 países miembros de la OCDE, en este último informe participaron otros 27, entre ellos Argentina. Para nuestro país no fue la primera vez, ya que había sido incluido en la primera edición de este ya clásico informe internacional en el año 2000.

En el informe de 2006 Argentina quedó por detrás de Uruguay, Chile, México y apenas por delante de Brasil y Colombia en dos de los tres dominios (matemáticas y ciencias) ya que en lectura estos últimos se ubicaron por delante de nuestro país.

En la evaluación general nuestra posición fue muy deficiente. Sobre 57 países, en matemáticas quedamos en el puesto 52 , en lectura 53 y en ciencias 51 , no siendo este dato banal si lo pensamos en el contexto del desarrollo social. Por ejemplo, Grossman y Kaestner en su exhaustiva revisión colocan a los años de escolarización formal como el indicador más fiable de correlación con buena salud ${ }^{1}$

La educación es uno de los mejores determinantes de la salud e incluso el único, sirviendo a todos los demás como medida de clase social. La educación, esencialmente reduce la desigualdad y la pobreza. Pensar en un país sano, es pensar en un país que trabaja y produce, y un país que produce, obviamente, crece. $\mathrm{El}$ desarrollo sostenible de un país tiene en la educación a su principal aliado como camino hacia la igualdad y la justicia.

Durante 2005 y con el fin de reducir las inequidades en salud y crear políticas basadas en evidencia, la Organización Mundial de la Salud (OMS) creó la Comisión para los Determinantes Sociales de la Salud ${ }^{2}$. Desde este evento, el origen social de la enfermedad ha estado más que nunca en la agenda pública.

El término "determinantes de salud" fue introducido en los años 70 como parte de una crítica a la visión que imperaba en aquel momento en la salud pública. Sin embargo, la evidencia la dieron los datos aportados por el Estudio Whitehall, que desde su primera publicación pusieron de manifiesto la importancia del concepto "gradiente social" medido en términos de ingresos económicos según clase social en la morbi-mortalidad de la población británica.
Hoy la salud pública se piensa como algo que concierne más a las políticas sociales y a los determinantes sociales que a los servicios de salud y los resultados de la enfermedad.

¿Cómo se relacionan los "determinantes" con la vida de las personas? A través de la posición social que condiciona una jerarquía social.

Veamos un ejemplo: en auto podríamos cubrir la distancia entre los barrios Recoleta y La Boca (barrios de la Ciudad de Buenos Aires) en aproximadamente 20 minutos. Conocemos las características socioeconómicas de los habitantes de estos dos barrios de Buenos Aires. ¿Cual será la brecha en expectativa de vida entre ellos?

Para el caso, en Washington DC (USA) se han medido expectativas de vida con una diferencia de 17 años según el barrio en que una persona vive $e^{4}$. Existe muchísima literatura sobre inequidad y salud, a pesar de eso hay muy poco escrito sobre como reducirla.

Hoy sabemos que los factores biológicos y sociales, a través de la vida y en forma independiente $\mathrm{y} / \mathrm{o}$ acumulativa pueden influenciar la salud y la enfermedad en la vida adulta. Muchos investigadores en salud sostienen que la posición social, y a través de ella en "como se vive", es la causa fundamental de la salud², encontrándose ésta en estrecha relación con la educación.

En la época que vivimos la mayor parte de los problemas de salud que sufrimos pueden ser aliviados educando a la población. La carga social y económica que imponen las lesiones de tránsito, las conductas de riesgo, la alimentación inadecuada o el sexo inseguro, podrían ser aliviadas si se implementan acciones de educación y promoción en salud.

\section{Pensando el futuro de Argentina}

Argentina es asimétrica en todos sus aspectos. Los niveles de ingreso per cápita, las condiciones de saneamiento o la proporción de la población dependiente de un deficiente sistema público de salud así lo demuestran. Conocemos esto desde hace tiempo.

La educación en general y la educación en salud de nuestra población en particular, representan una estrategia válida para lograr una disminución de esa asimetría. Hace mucho tiempo se dijo que la educación debe garantizar la promoción de los valores de libertad y solidaridad, sin embargo debemos ser conscientes de que estos valores se enseñan.

¿Cuánto de la importancia de un país o de su capacidad de influir o de competir en el mundo está relacionados con como se educan sus niños?

* Medico. Master en Gerontología y candidato a Doctor en Medicina por la Universidad de Salamanca. España. diegobernardinimd@ hotmail.com

** Politólogo. Master en Estudios Latinoamericanos y candidato a Doctor en Ciencias Políticas por la Universidad de Salamanca. España. 
Además de la educación "clásica", ¿que otras educaciones deberían recibir nuestros escolares de cara a los desafíos del mundo que nos toca vivir? Por ejemplo, laa carencia de educación sexual podría implicar la aparición de algunos emergentes prevenibles como la epidemia del SIDA y otras enfermedades de transmisión sexual, los embarazos no deseados y/o la violencia sexual, todos ellos con costos humanos y económicos.

¿Qué hay por ejemplo de las lesiones traumáticas no intencionales relacionadas al tránsito vehicular? (antes llamadas "accidentes de tránsito" y que por cierto tienen muy poco de accidentales). Siendo este tipo de lesión la principal causa de muerte de nuestros jóvenes de 18 a 35 años, sobran motivos para incluir a la educación vial como una prioridad en Argentina.

Nuestro país es destacadamente rico en recursos naturales renovables. Una importante inversión en nuestro futuro sería introducir contenidos de educación ambiental con el objetivo de poder disfrutar dichos recursos por más tiempo, y para que en pocos años actuemos con conciencia ciudadana de la necesidad del ahorro energético, del reciclado de la basura o del mejor aprovechamiento del agua.

Pensar en estos aspectos es una forma de tener en cuenta nuestra realidad como forma de ir moldeando la sociedad que queremos para nuestro futuro.

Los españoles, los franceses y los alemanes hace tiempo que están debatiendo su reforma educativa. Sin ir más lejos, los resultados para España de este último informe PISA fueron catastróficos ya que han retrocedido en los tres dominios medidos (matemáticas, lectura y ciencias) en relación al informe previo de 2003 y en relación a la media de la OCDE.

La consecuencia de la difusión de estos datos fue un intenso debate y una crítica despiadada a las autoridades por parte de los medios de prensa nacionales.
Muchas veces tomamos como ejemplos de desarrollo a los países nórdicos y vemos como en ellos estas materias forman parte de sus programas educativos hace décadas, con el consiguiente resultado efectivo. Por eso cabe preguntarse ¿por qué tardamos tanto en implementar planes ya consolidados en otras sociedades? Por ejemplo, ¿por qué debió pasar tanto tiempo en Argentina hasta que se implementara la obligatoriedad de circular por las rutas con la "luces bajas" encendidas durante todas las horas del día? Vale mencionar que nuestro país vecino Uruguay, ya lo había implementado casi tres años antes.

Hoy Argentina tiene la oportunidad de poder recuperar una parte importante de ese escalón que descendió con la última crisis del 2001.

\section{Conclusiones}

Desde las formas más básicas, la educación tiene una dimensión inmaterial en el corto plazo y una gravitación fundamental como activo económico. Incide en la salud, en el bienestar, en la cultura, en el producto bruto interno; y es un activo que genera ideas y empleos. Las oportunidades existen, pero sacarán mayores ventajas de ellas quienes sepan aprovecharlas de mejor forma. Consideramos que en la actualidad el debate sobre la educación debería ser el primero de una serie incluya a la educación para la salud, la educación vial, la educación ambiental o la simple y no menos importante educación soberana de nuestros niños como ciudadanos con derechos y obligaciones.

Lo que si debe ser claro que en este debate se empieza a discutir qué clase de país queremos para nuestro futuro.

Al presente podemos afirmar que el futuro pertenecerá a aquellos que mejor sepan crear, transmitir y aplicar el conocimiento, y el primer paso de esto es la escuela. En ella es donde empieza el ejercicio de la responsabilidad individual.

Hace tiempo que los mensajes y las políticas no se corresponden con la realidad demográfica. ¿No será hora de dejar de mirar al costado? ¿Necesitaremos más evidencia para generar cambios? Asistimos a una "ventana de oportunidad", pero como toda ventana, en algún momento se cierra...

Referencia

Recibido el 02/09/09 y aceptado el 26/01/09.

1. Grossman M y col.: Effects of education on health; in Behrman JR, Stacey N., (eds): The Social benefits of Education. Ann Harbor, University of Michigan Press, 1997, pp 69-123. 2. World Health Organization. Commission on Social Determinants of Health - Final Report. Closing the gap in a generation: Health equity through action on the social determinants of health. Disponible en URL: http://whqlibdoc.who.int/publications/2008/9789241563703_eng.pdf (útimo acceso 26/01/09).

nants of health. Disponible en URL: http://whqlibdoc.who.int/publications/2008/9789241563703_eng.pdf (útimo acceso $26 / 1$.

3. Marmot M y col. Health inequalities among British Civil Servants: the Whitehall II study. Lancet 1991; $337: 1387-1393$.
4. Murray C y col. (2006) Eight Americas: Investigating mortality disparities across races, counties, and race counties in the United States. Plos Med 3(9):e260.DOI:10.1371/Journal.pmed.0030260

\section{AL LECTOR}

Estimado Suscriptor de Evidencia

Como es de público conocimiento, durante 2008 hemos sufrido un incremento significativo en todos nuestros costos de producción de la publicación.

Es por ello que para poder continuar brindando a nuestros lectores un producto de la misma calidad y contenidos editoriales sin trasladar este incremento inflacionario del $25 \%$ en forma completa, le comunicamos que a partir de 2009, EVIDENCIA se publicará en forma trimestral en vez de bimestral (enero, abril, julio y octubre) y cada uno de los números contará con mayor contenido.

De este modo, el aumento de la suscripción anual será solamente del $15 \%$.

Esperamos poder seguir contando con su apoyo para poder continuar con la publicación.

Lo saludamos muy atentamente.

Evidencia 